\title{
Emerging Dimensions in the Energy Harvesting
}

\author{
${ }^{1}$ Alok Jain, ${ }^{2}$ Mrs. Suman Bhullar \\ ${ }^{I}$ M.E. (Psed), Thapar University, Patiala-147004, Punjab (India). \\ ${ }^{2}$ Assistant Professor, Thapar University, Patiala-147004, Punjab (India).
}

\begin{abstract}
Energy is everywhere in the environment surrounding us i.e. it is available in the form of thermal energy, light (solar) energy, wind energy, and mechanical energy etc. However, the energy from these sources is often found in such minute quantities that it cannot supply adequate power for any viable purpose. In fact, it has not been possible to capture such energy sufficiently to perform any useful work. But now the scenario is changing due to new technologies on energy harvesting being developed and used.

Energy harvesting is a process of capturing minute amounts of energy from one or more of these naturally-occurring energy sources, accumulating them and storing them for later use. Energy-harvesting devices efficiently and effectively capture, accumulate, store, condition and manage this energy and supply it in a form that can be used to perform a helpful task.

Though the energy harvesting has been a phenomenon in solar and wind energy areas, now time has come to venture into other energy areas and to improve our harvesting capabilities, proliferate harvesting devices, and apply them to useful systems. This paper reviews significance and applications areas of the energy harvesting. It also visits the design considerations and different types of energy harvesting systems.
\end{abstract}

\subsection{Introduction To Energy Harvesting}

\section{Introduction}

Energy harvesting (also known as power harvesting or energy scavenging) is the process by which energy is derived from external sources (e.g., solar power, thermal energy, wind energy, salinity gradients, and kinetic energy), captured, and stored. Frequently, this term is applied when speaking about small, wireless autonomous devices, like those used in wearable electronics (where energy harvesting devices can power or recharge cell phones, mobile computers, radio communication equipment, etc.) and wireless sensor networks.[1]

\subsection{Overview}

Traditionally, electrical power has been generated in large, centralized plants powered by fossil fuels, nuclear fission or flowing water. Large-scale ambient energy, such as sun, wind and tides, is widely available but technologies do not exist to capture it with great efficiency. Energy harvesters currently do not produce sufficient energy to perform mechanical work, but instead provide very small amount of power for powering low-energy electronics. While the input fuel to large scale generation costs money (oil, coal, etc.), the "fuel" for energy harvesters is naturally present and is therefore considered free. For example, temperature gradients exist from the operation of a combustion engine and in urban areas,there is also a large amount of electromagnetic energy in the environment because of radio and television broadcasting.

Advanced technical developments have increased the efficiency of devices in capturing trace amounts of energy from the environment and transforming them into electrical energy. In addition, advancements in microprocessor technology have increased power efficiency, effectively reducing power consumption requirements. In combination, these developments have sparked interest in the engineering community to develop more and more applications that utilize energy harvesting for power.

Energy harvesting from a natural source where a remote application is deployed, and where such natural energy source is essentially inexhaustible, is an increasingly attractive alternative to inconvenient wall plugs and costly batteries. This essentially free energy source, when designed and installed properly, is available maintenance-free and is now available throughout the lifetime of the application. Such systems can be more reliable than wall plugs or batteries.

In addition, energy harvesting can be used as an alternative energy source to supplement a primary power source and to enhance the reliability of the overall system and prevent power interruptions. 


\subsection{Different Sources of Energy Harvesting}

\section{Sources Of Energy Harvesting}

Various energy sources (natural sources such as wind, solar, water flow, ocean currents etc.) are kept beyond the scope of this paper due to their significant technological progress.

The energy can be captured from a variety of sources deemed wasted or otherwise unusable for any practical purpose. The process, also known as energy scavenging, captures residual energy as a by-product of a natural environmental phenomenon or industrial process and is therefore considered "free energy." More often than not, this residual energy is released into the environment as waste.

\begin{tabular}{|c|c|c|c|}
\hline Energy Source & Characteristics & Efficiency & Harvested Power \\
\hline Light & $\begin{array}{l}\text { Outdoor } \\
\text { Indoor }\end{array}$ & $10 \sim 24 \%$ & $\begin{array}{l}100 \mathrm{~mW} / \mathrm{cm}^{2} \\
100 \mu W / \mathrm{cm}^{2}\end{array}$ \\
\hline Thermal & $\begin{array}{l}\text { Human } \\
\text { Industrial }\end{array}$ & $\begin{array}{l}-0.1 \% \\
-3 \%\end{array}$ & $\begin{array}{l}60 \mu W / \mathrm{cm}^{2} \\
\sim-10 \mathrm{~mW}^{2} \mathrm{~cm}^{2}\end{array}$ \\
\hline Vibration & $\begin{array}{l}-\mathrm{Hz}-\text { human } \\
-\mathrm{kHz}-\text { machines }\end{array}$ & $25-50 \%$ & $\begin{array}{l}-4 \mu W / \mathrm{cm}^{2} \\
-800 \mu W / \mathrm{cm}^{3}\end{array}$ \\
\hline RF & $\begin{array}{l}\text { GSM } 900 \mathrm{MHz} \\
\text { WiFI }\end{array}$ & $-50 \%$ & $\begin{array}{l}0.1 \mu W / \mathrm{cm}^{2} \\
0.001 \mu W / \mathrm{em}^{2}\end{array}$ \\
\hline
\end{tabular}

Table 1 shows different sources of energy harvesting

Examples include mechanical energy resulting from vibration, stress and strain, thermal energy from heat escaped from furnaces, combustion engines and other heating sources as shown in table 1. Other sources are biological, solar energy from all forms of light sources; electromagnetic energy captured via inductors, coils and transformers; wind and fluid energy resulting from air and liquid flow; chemical energy from naturally recurring or biological processes; and huge amounts of RF energy in the environment because of ubiquitous radio transmitters and television broadcasting.

1. Mechanical energy resulting from vibration, stress and strain. When a piezoelectric transducer is stressed mechanically by a force, its electrodes receive a charge that may be collected, stored and delivered to power circuits or processors. MEMs vibrational energy harvesters operating in the frequency domain between 150 and $1000 \mathrm{~Hz}$ are particularly well suited to convert vibrations from machines, engines and other industrial appliances into electricity.

2. RF energy harvesting converts radio waves into DC power. This is accomplished by receiving radio waves with an antenna, converting the signal, and conditioning the output power. In cities and very populated areas there is a large number of potential RF sources including broadcast radio and television, mobile telephony, wireless networks, etc.

At $100 \mathrm{~m}$ from the typical $100 \mathrm{~W}$ cellular base station, for example, there is about $800 \mu \mathrm{W} / \mathrm{m}^{2}$ of energy available. Your GSM handset is good for about $0.1 \mu \mathrm{W} / \mathrm{cm}^{2}$.

3. Solar energy from light (using photovoltaic devices) once the exclusive domain of space science, has now come down in price to where solar powered watches and phones are available. Approximate available power: indoor light $10 \mu \mathrm{W}$ or less, outdoors $0.10-15 \mathrm{~mW} / \mathrm{cm}^{2}$.

4. Temperature differentials (via thermoelectric generators or thermopiles attached to a heat generating source, such as an HVAC duct, furnaces, combustion engines etc.) Heat from industrial equipment has potential electrical power on theorder of $1-10 \mathrm{~mW} / \mathrm{cm}^{2}$; the human body can generate $15-30 \mu \mathrm{W} / \mathrm{cm}^{2}$ as in figure shown1.[2]
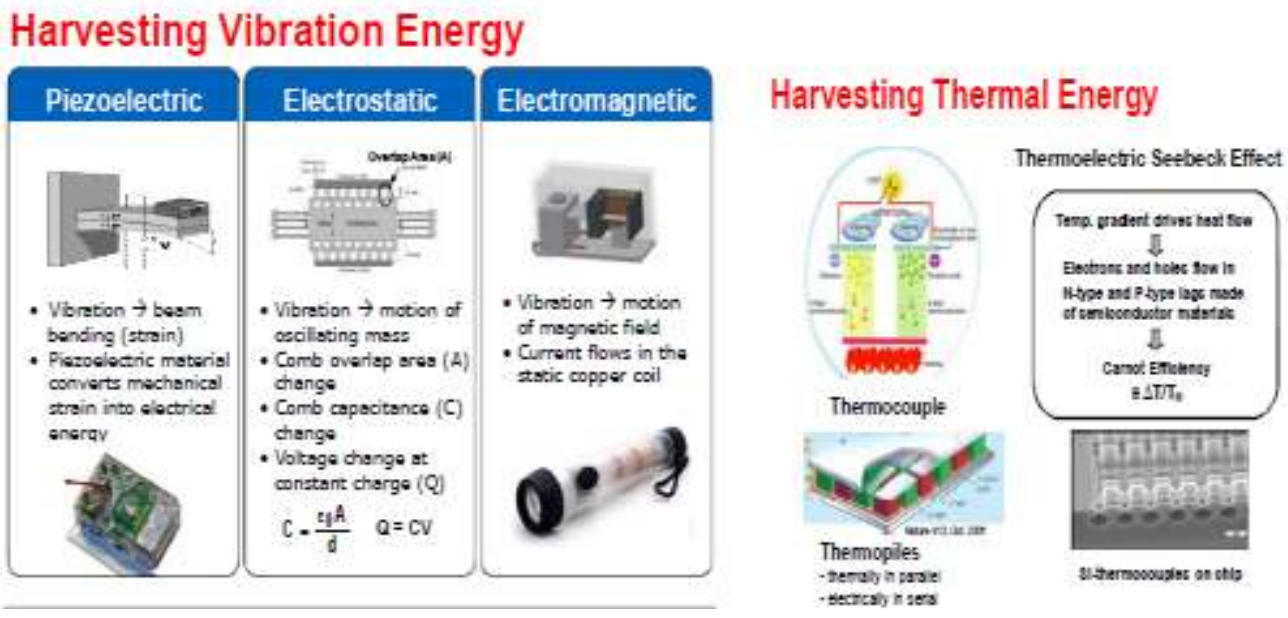
Tree Energy Harvesting
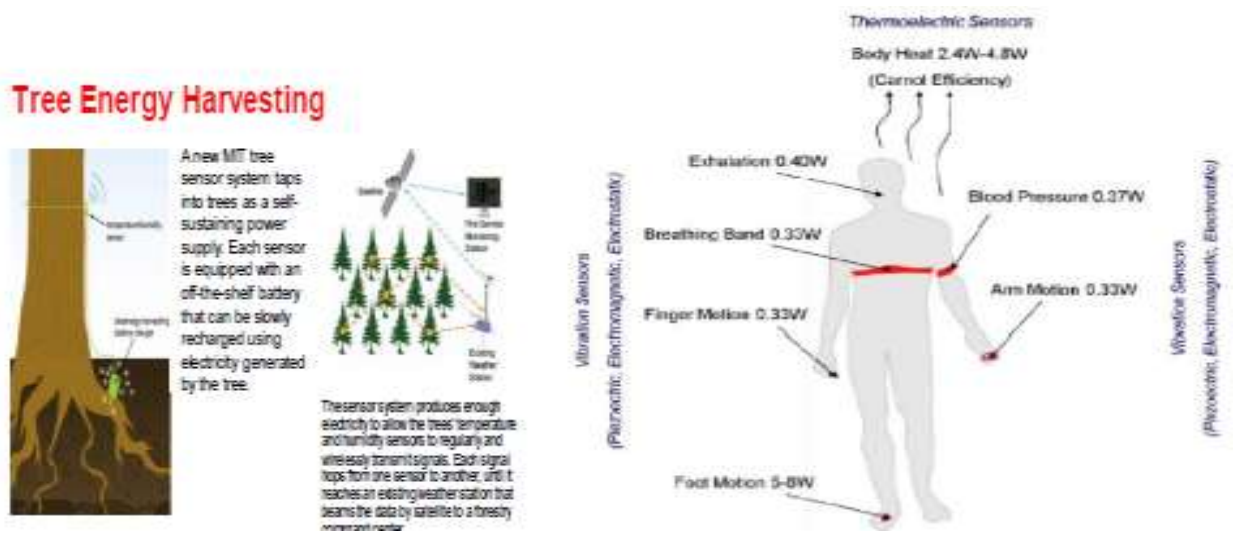

Figure 1 shows common sources of energy harvesting

\section{Energy Harvesting System}

\subsection{Components Of Energy Harvesting System}

An energy harvesting system generally requires an energy source such as vibration, heat, light or air flow and three other key electronic components, as shown in figure 2 including:-

- An Energy conversion device such as a piezoelectric element that can translate the energy into electrical form;

- An energy harvesting module that captures stores and manages power for the device;

- An End application such as a ZigBee[3]-enable wireless sensor network or control and monitoring devices.

\subsection{Operation Block Diagram}

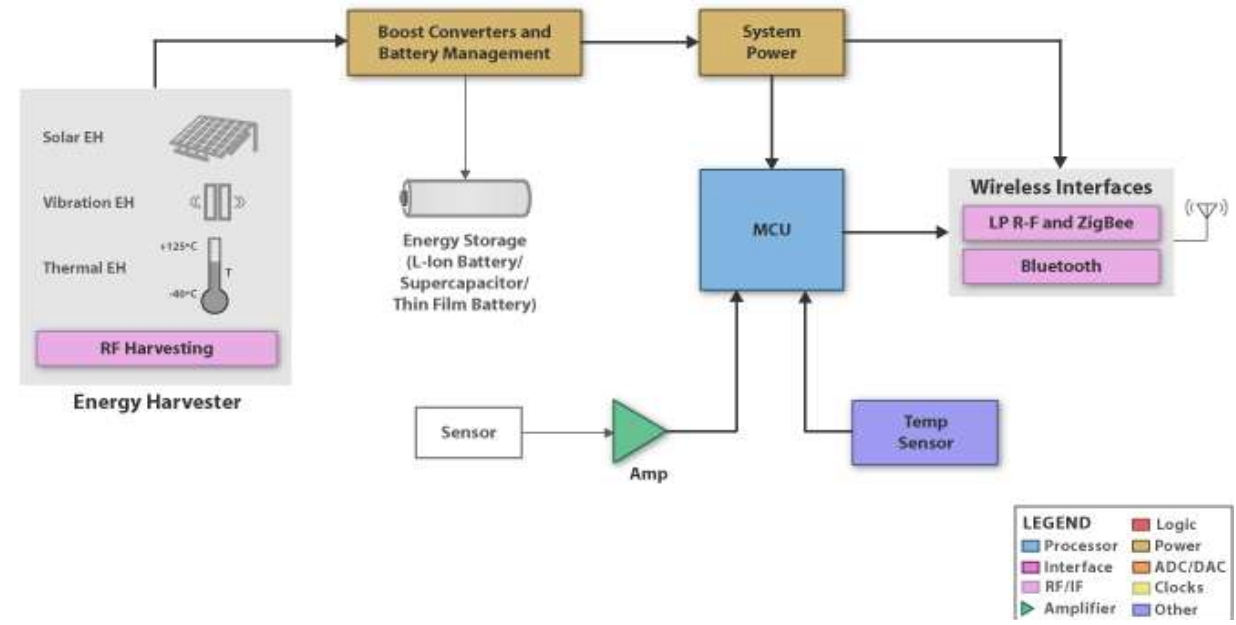

Figure 2 shows the block diagram of energy harvesting system

\section{- Design Considerations:}

An Energy Harvesting System consists of an Energy Harvester Module and a processor/transmitter block. The Energy Harvester module captures milli-watts of energy from light, vibration, thermal or biological sources. A possible source of energy also comes from RF such as emitted from cell phone towers. The power is then conditioned and stored within a battery, an efficient quick charging capacitor or one of the newly developed thin film batteries. The system is then triggered at the required intervals to take a sensor reading, through a low power system. This data is then processed and transmitted to the base station. This kind of EH System eliminates the dependency of the system on battery power and reduces the need toservice the system as shown in figure.[4]

\section{- Accumulating energy:}

Energy can also be harvested to power small autonomous sensors such as those developed using MEMS[5] (Micro Electro-Mechanical System) technology. These systems are often very small and require little power, but their applications are limited by the reliance on battery power. Scavenging energy from ambient vibrations, wind, heat or light could enable smart sensors to be functional indefinitely. 
Typical power densities available from energy harvesting devices are highly dependent upon the specific application (affecting the generator's size) and the design itself of the harvesting generator. In general, for motion powered devices, typical values are a few $\mu \mathrm{W} / \mathrm{cm}^{3}$ for human body powered applications and hundreds of $\mu \mathrm{W} / \mathrm{cm}^{3}$ for generators powered from machinery.

\section{- Storage of power:}

In general, energy can be stored in a capacitor, super capacitor, or battery. Capacitors are used when the application needs to provide huge energy spikes. Batteries leak less energy and are therefore used when the device needs to provide a steady flow of energy.

\section{- Use of the power:}

Current interest in low power energy harvesting is for independent sensor networks. In these applications an energy harvesting scheme puts power into stored into a capacitor then boosted/regulated to a second storage capacitor or battery for the use in the microprocessor. The power is usually used in a sensor application and the data stored or is transmitted possibly through a wireless method.

\subsection{Biomechanical Harvesting}

\section{Types Of Energy Harvesting}

Biomechanical energy harvesters are also being created. One current model is the biomechanical energy harvester of Max Donelan which straps around the knee.[6] Devices as this allow the generation of 2.5 watts of power per knee. This is enough to power some 5 cell phones. Biomechanical Energy Harvesting: Generating electricity during walking with minimal user effort-Biomechanical energy harvesters have been developed that generates electricity during human walking with little extra effort.

Unlike conventional human-powered generators that use positive muscle work, our technology assists muscles in performing negative work, analogous to regenerative braking in hybrid cars, where energy normally dissipated during braking drives a generator instead. The energy harvester mounts at the knee and selectively engages power generation at the end of the swing phase, thus assisting deceleration of the joint. Test subjects walking with one device on each leg produced an average of 5 watts of electricity, which is about 10 times that of shoe-mounted devices. The cost of harvesting isthe additional metabolic power required to produce 1 watt of electricity which is less than one-eighth of that for conventional human power generation. Producing substantial electricity with little extra effort makes this method well-suited for charging powered prosthetic limbs and other portable medical devices.

\subsection{Piezoelectric Energy Harvesting}

The concept of piezoelectricity was first discovered by Pierre and Jacques Curie in 1880 in quartz and in Rochelle salt. When these materials are subjected to mechanical pressure, electrical potential will appear on the faces of the crystals. When the crystals are compressed, it forces negative ions within each unit cell of the crystal to rearrange itself. This leads the polarization of the unit cells and on a larger scale, the appearance of an electrical potential difference on the faces of the crystals. The piezoelectric effects are found in several types of crystals and also in some ceramics. These materials are used in transducers, ultrasonic receivers, and in microphones. Application of stress to a piezoelectric crystal generates an equivalent electric charge; conversely applying a voltage induces a shape change.

As-

1. Haussler [7] proposed an implantable physiological power supply using PVDF films.

2. Umeda[8] looked at using impact energy from a steel ball dropped onto a plate with a piezoelectric material attached.

3. Elvin [9] theoretically and experimentally investigated the use of self-powered PVDF strain sensors.

The piezoelectric effect converts mechanical strain into electric current or voltage. This strain can come from many different sources. Human motion, low-frequency seismic vibrations, and acoustic noise are everyday examples.

Most piezoelectric electricity sources produce power on the order of milliwatts, too small for system application,butenoughforhand-helddevicessuchassomecommerciallyavailableself-winding wristwatches. One proposal is that they are used for micro-scale devices, such as in a device harvesting micro-hydraulic energy. In this device, the flow of pressurized hydraulic fluid drives a reciprocating piston supported by three piezoelectric elements which convert the pressure fluctuations into an alternating current.

The use of piezoelectric materials to harvest power has already become popular. Piezoelectric materials have the ability to transform mechanical strain energy into electrical charge. Piezo elements are being embedded in 
walkways [10] [11] [12] to recover the "people energy" of footsteps. They can also be embedded in shoes[13] to recover "walking energy" and also the Electro active polymers (EAPs)[14] has been proposed for harvesting energy as shown in figure 3. These polymers have a large strain, elastic energydensity, and high energy conversion efficiency.The total weight of systems based on EAPs is proposed to be significantly lower than those based on piezoelectric materials.

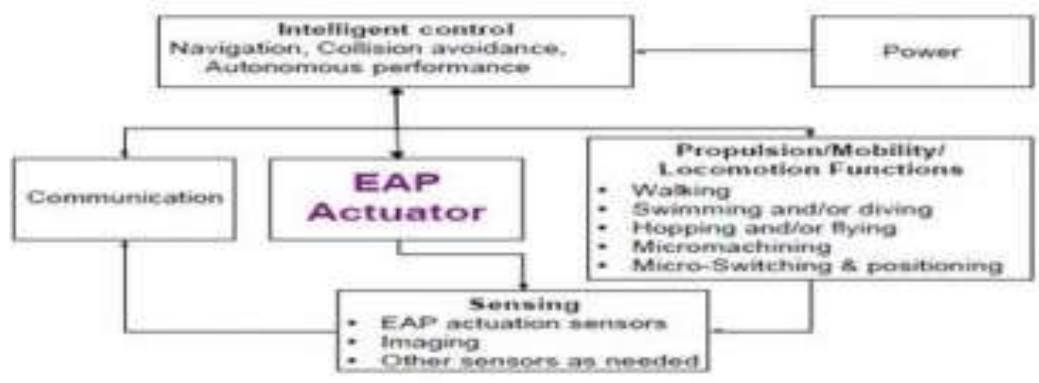

Figure 3 shows EAP for energy harvesting

\subsubsection{Energy Harvested From Automobile Traffic On Highways}

The field of engineering known as piezo-electric, and the tiny land of Israel and the coastal areas are not easy to get natural sources of energy such as oil and gas fields, many Israeli companies innovative scientific technologies in pursuit of the 'brass ring' success. OnesuchcompanyInnowattech, patents for a unique approach to cleaning conventional urban planning center - with the possibility of retained and re-use the energy of the roads or highways, railways, runways and even sidewalks.

The system is called for IPEG Innowattech Piezoelectric Generator developed by (Innowattech[15]), with significant potential for energy normally wasted by conventional vehicles, which abound in the urban centers of normal life: cars, buses, trains, etc. The design principles include weight changes, movement, vibration and temperature, the concept of using piezoelectric generator designed specifically for use in roads, places of railways, runways, etc. as shown in figure 4 .

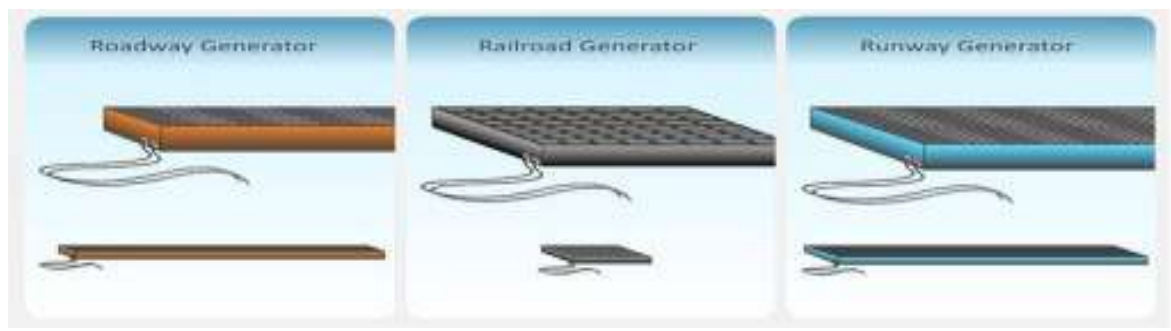

Figure 4 shows piezoelectric generator designed specifically for use in roads, places of railways, runways

The general concept is to capture or collect, store and reuse parasitic mechanical energy; Piezo-electric effect of converting mechanical "strain" energy into electric current or voltage. Constitutive relations for piezoelectric materials based on the total strain in the actuator, the amount of mechanical strain-induced stress, thermalstraincausedbythedifferenceintemperature, aswellasbringingacontrolled deformation caused by the electric voltage - all included in the piezo-electric effect. (Strain is a physical dimension change in homogeneous materials, the use of force in the stress and Young Modulousmaterial; Strain = Stress / Modulous.)

Piezoelectric generators are designed for different sizes from a few centimeters to large surfaces, as well as embedded under the asphalt topping layer. Parasitic energy busy roads, railway track beds and runways the airport, only about settlements, has the potential to convert into electrical energy, which can be delivered to customers or the national electric network. Normal car bearing urban roads may be technically described as a visco-elastic-plastic material; the elasticity is the most important parameter, and physical characteristics. As the car moves along the road, the vertical displacement takes place under the tires, which transmit the weight of the vehicle on the road - the reaction was heat. With piezoelectricgenerators embedded in road materials, part of the energy expended on deformation of the road can be converted into electrical energy (piezo-electric effect) and to restore, rather than simply wasted as heat.

\subsubsection{Active Control Based Energy Harvesting For Battery-Less Wireless Traffic Sensors}

A novel battery-less wireless sensor is developed that can be embedded in the road and used to measure traffic flow rate, speed and vehicle weight. Compared to the ubiquitous inductive loop based traffic 
sensor, the new sensor is expected to provide increased reliability, easy installation and low maintenance costs. Energy to power this sensor is harvested from the short duration vibrations that results when an automobile passes over the sensor. Since all of the earlier work in literature on vibration energy harvesting has focused on continuous sources of vibration, it focuses on short duration vibrations and on developing low power control algorithms that can be implemented on the sensor using an analogcircuit as shown in figure 5.

To this effect it develops and compares three control algorithms:-

- "Fixed threshold switching",

- "Maximum Voltage switching" and,

- "Switched Inductor" for maximizing this harvested energy.

The "Switched inductor" algorithm is shown to be the most effective at maximizing harvested energy.

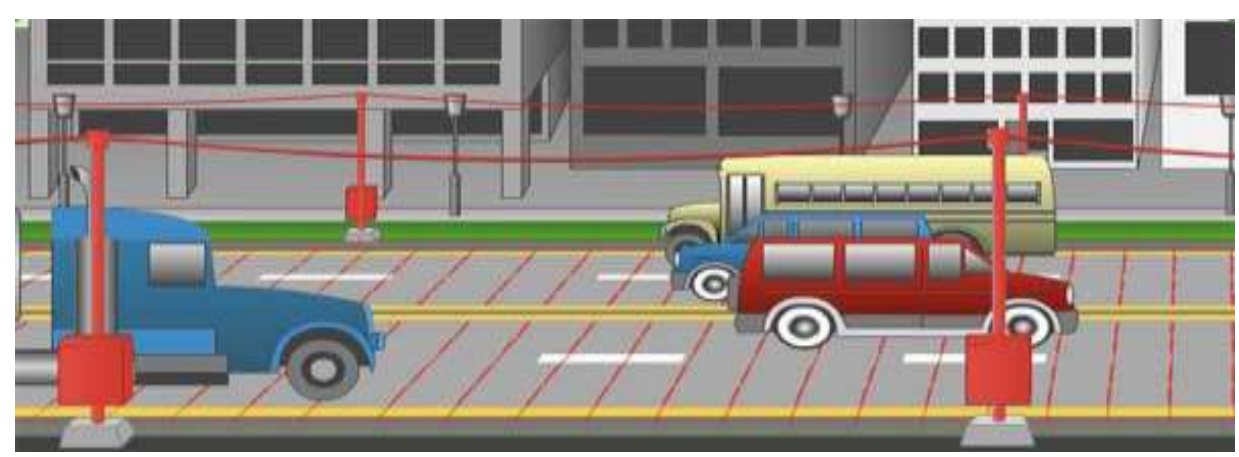

Figure 5 shows a novel battery-less wireless sensor embedded in the road

\subsubsection{Review of current traffic sensors}

Transportation agencies all around the country monitor traffic flow rates on most major highways. For example, the Minnesota Department of Transportation (MnDOT[16]) uses inductive loop detectors to monitor the flow rates at over 6000 points in the Minneapolis/St. Paul metro area. The flow rate information from such sensors is used to control ramp meters, identify congestion points, detect incidents and for a number of other applications. Despite various new technologies for detecting vehicles such as image processing based detectors and sound based systems inductive loop detectors remain the most widely used technology for detecting and counting vehicles due to their high accuracy. An inductive loop detector consists of a big loop of metallic coil buried in the lane. This loop is connected to astation which powers the loop and processes the information obtained from the loop to determine if a vehicle passes over the sensor. Despite their popularity, inductive loops are far from perfect and there has been considerable work to improve traffic detection using better models, better filtering technology and by using better identification techniques such as Fuzzy Logic.[17]

\subsubsection{Proposed battery-less traffic sensors(hardware)}

A developed novel battery-less wireless traffic sensor, which like the inductive loop does not use complex image processing or audio processing techniques and would hence provide the same level of accuracy. Further this sensor has smaller dimensions and can be installed with much lower traffic disruptions. This is especially true because the sensor does not need a power source and power lines do not need to berun to the sensor. When an automobile passes over the sensor, a RF pulse is transmitted wirelesslyto the station. By using different frequencies, several sensors can transmit to the same station. Owing to the battery-less and wireless nature of the sensor high reliability and low maintenance are to be expected. Further it is likely that the sensor can measure the number of axles and weight of passing vehicle in addition to measuring the traffic flow rate. The proposed sensor is based on the principle of harvesting energy to enable wireless transmission of signals. Sodanaet. Al[18]provides a good review of vibration energy harvesting techniques which focus predominantly on harvesting energy from a continuous sourceof vibration. When a vehicle passes over the sensor however, it would give rise to short term vibration in the mechanical structure.

\subsubsection{Some Examples Of Piezoelectric Energy Harvesters:}

1. Energy harvesting for robots.

2. Energy harvesting backpack.

3. Harnessing vibrations from raindrops.

4. Flapping leaf generator for wind energy harvesting.

5. Piezoelectric kinetic energy harvester for mobile phones. 
6. Breakthroughs with sensing in the human body.

\subsection{Pyroelectric Energy Harvesting}

The pyroelectric effect converts a temperature change into electric current or voltage. It is analogous to the piezoelectric effect, which is another type of ferroelectric behavior. Like piezoelectricity, pyroelectricityrequires time-varying inputs and suffers from small power outputs in energy harvesting applications. Pyroelectric materials are stable up to $1200 \mathrm{C}$ or more, enabling energy harvesting from high temperature sources and thus increasing thermodynamic efficiency. There is a pyroelectric scavenging device that was recently introduced, which doesn't require time-varying inputs. The energy-harvesting device uses the edge-depolarizing electric field of a heated pyroelectric to convert heat energy into mechanical energy instead of drawing electric current off two plates attached to the crystal-faces. Moreover, stages of the novel pyroelectric heat engine can be cascaded in order to improve the Carnot efficiency.[19]

\subsection{Thermo Electrics}

In 1821, Thomas Johann Seebeck discovered that a thermal gradient formed between two dissimilar conductors produces a voltage. At the heart of the thermoelectric effect is the fact that a temperature gradient in a conducting material results in heat flow; this results in the diffusion of charge carriers.

The flow of charge carriers between the hot and cold regions in turn creates a voltage difference. In 1834, Jean Charles AthanasePeltier discovered that running an electric current through the junction of two dissimilar conductors could, depending on the direction of the current, cause it to act as a heater or cooler. The heat absorbed or produced is proportional to the current, and the proportionality constant is known as the Peltiercoefficient. Today, due to knowledge of theSeebeck and Peltier effects, thermoelectric materials can be used as heaters, coolers and generators (TEGs).[20]

\subsubsection{Advantages to thermo electrics:}

- TellurexCorporation[21] (a thermoelectric production company) claims that thermo electrics are capable of over 100,000 hours of steady state operation.

- Thermo electrics contain no materials that must be replenished.

- Heating and cooling can be reversed.

- One downside to thermoelectric energy conversion is low efficiency (currently less than $10 \%$ ).

Miniature thermocouples have been developed that convert body heat into electricity and generate $40 \mu \mathrm{Watt} 3 \mathrm{~V}$ with a 5 degree temperature gradient, while on the other end of the scale, large thermocouples are used in nuclear RTG batteries. Practical examples are the finger-heart rate meter by the Holst Centre and the thermo-generators by the FraunhoferGesellschaft.[22]

\subsection{Electrostatic (Capacitive) Energy Harvesting}

It refers to capacitive harvesting. Electrostatic generators are widely used though they are not as popular as piezoelectric or thermoelectric. Electrostatic (capacitive) energy harvesting is based on the changing capacitance of vibration-dependent variable capacitors called varactors. Vibrations separate the plates of an initially charged varactor, and mechanical energy is converted into electrical energy. To put it more precisely, with electrostatic generators, mechanical forces are employed to do work against the attraction of differently charged parts, so they are mechanically variable capacitors with plates separated by the movement of the source. They are either switched (switched charge or potential) or continuous. All electrostatic generators require potential and inorganic or organic electrets are one way of providing this.

Electrets have a permanent charge buried in the dielectric. For example, PVDF and certain inorganic oxides act as electrets and some are used as such in microphones. An active charge system could permit tuning to duty cycle. For example of an electrostatic energy harvester with embedded energy storage is the M2E Power Kinetic Battery. [23]

\subsection{Blood Sugar Energy Harvesting}

The energy harvesting i.e. through the oxidation of blood sugars. These energy harvesters are called biofuel cells.[24] They could be used to power implanted electronic devices (e.g., pacemakers, implanted biosensors for diabetics, implanted active RFID devices, etc.). At present, the Minteer Group of Saint Louis University has created enzymes that could be used to generate power from blood sugars. [25]

\subsection{Tree Metabolic Energy Harvesting}

Tree metabolic energy harvesting is a type of bio-energy harvesting. Voltree[26] has developed a method for harvesting energy from trees. These energy harvesters are being used to power remote sensors and 
mesh networks as the basis for a long term deployment system to monitor forest fires and weather in the forest. Their website says that the useful life of such a device should be limited only by the lifetime of the tree to which it is attached as shown in figure 6.

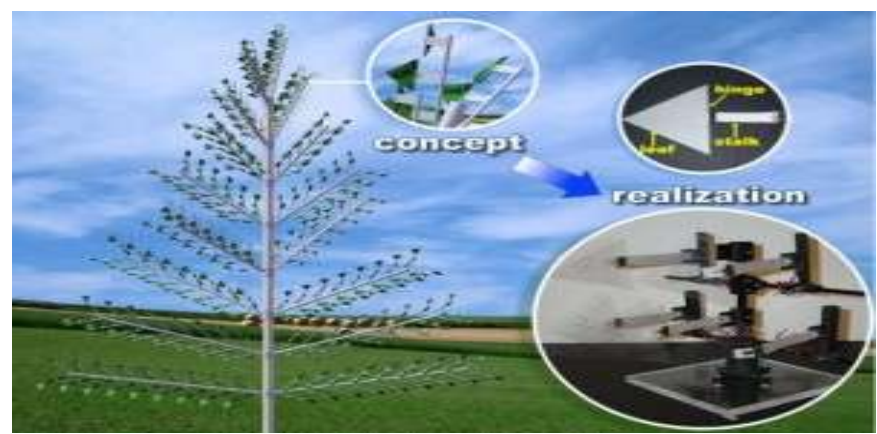

Figure 6 shows tree metabolic energy harvesting

\subsection{Significance}

\section{Significance Of Energy Harvesting}

Advanced technical developments have increased the efficiency of devices in capturing trace amounts of energy from the environment and transforming them into electrical energy. In addition, advancements in microprocessor technology have increased power efficiency, effectively reducing power consumption requirements. In combination, these developments have very wide interest in the engineering community to develop more and more applications that utilize energy harvesting for power.

\subsection{Characteristics Of Energy Harvesting Applications}

1. Ability to operate with lowest standby current to maximize storage of energy.

2. Consume lowest possible power when active.

3. Ability to turn on and turn off instantaneously.

4. Efficient operation with lowest duty cycle of active v/s standby modes.

5. Analog capability for sensor interfacing and measurements.

6. Ability to operate with a low voltage range.

7. Lowest leakage currents to maximize harvested energy.

\subsection{Energy Harvesting Applications}

Many real life applications using energy harvesting system power are now practical. Wireless sensor network systems such as ZigBee systems often benefit from energy harvesting power sources. For example, when a wireless node is deployed at a remote site where a wall plug or a battery is either unreliable or unavailable, energy harvesting can augment or supply power as shown in figure7.[27]

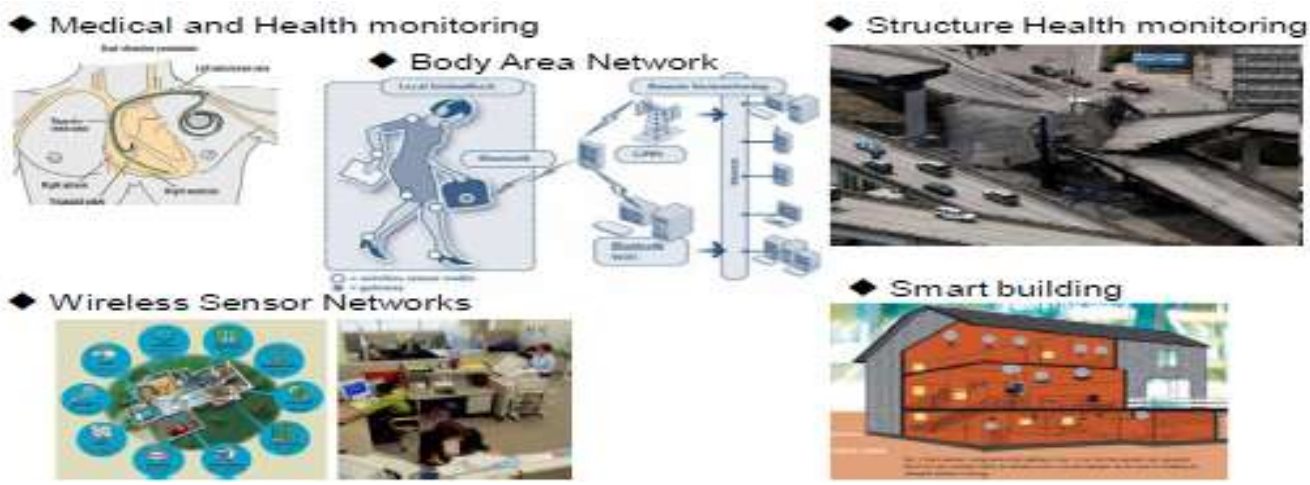

Figure 7 shows the applications of energy harvesting

* So one particular area that can be addressed with his low and no-power type of applications is like the office environment. Today, most offices are built of cubes, temporary arrangements of workspaces etc. Now when the office was constructed, there was a lot of permanent things like light switches, thermostats etc. 
In an environment where you could have sensors that took low or no power, you could distribute those to where the workplace is. So each individual cube could have its own thermostat. The light control could be there for that particular environment, and this allows a lot more flexibility in building utilization.

* Let's look at a couple of possible applications that are all new, and we depict here a patient in a hospital. And I think if anyone has been in a hospital or visited anyone in a hospital, you're aware that one of the great aggravations is that every hour or so you have a nurse show up and poke and prod and take measurements off you so they can keep an eye on your progress and your vital statistics, your vital signs. And imagine a sensor that could be just adhered to your skin, could run off your body heat, measure the types of data that people are looking for, your temperature, your blood pressure, your heart rate, your blood oxygen level, and transmit those wirelessly to the base of your bed or to a central station.

This not only improves the patient's comfort factor in the hospital and improves their recovery capabilities, but it's probably also going to make it possible to staff the hospital more efficiently. And you can imagine a single person could observe and monitor far more patient's than is currently needed to do the same job. So the ability with things like technology like this to extend the reach of technology into things like patient monitoring.

Of course, looking below that to the bridge, the patient doesn't necessarily have to be human. If you can imagine us taking a set of strain gauges and paint condition monitors and vibration monitors and attaching it to a structure like the Golden Gate Bridge. And to be able to, again, wirelessly correlate this data and observe the health of the structure and the use level of the structure, over time, it would help us to make sure that the thing remains safe and that we were able to maintain it at a minimal expense.

* Another application space that has lots of growth opportunities is actually the consumer space,especially centeredaround the home. We have previously talked about RFID and how that usesharvested energy to identify your vehicle when it's passing through a tollgate.

That sameapproach could be used for like controlling security gates, your garage, and if you look at that,then you no longer need that clicker that you associated with garage door openers and especiallythe management of that, making sure that it has fresh batteries and that you haven't lost it.

* Just as an example and we show a lot of them here with things that can be done as far assprinkler systems sensingwhen you need them; turn them off and connecting all thosemeasurements wirelessly to homecontrol units.

* We can just kind of narrow it down to one focus right now. Let's just talk about the lowly smokedetector. Now today's smoke detectoris meant to detect particles in the air and alert the sleeperor the occupants of the house to a problem, indicating if there's smoke there must be a fire.

Well,in a typical year, about 900 people in the United States die with smoke detectors installed in theirliving area. And the reason they die with even smoke detectors is because the smoke detectorsdon't sound, and the principal reason for them not sounding is they have lost power.

They eitherhave the battery disconnected or poorly connected. So why does that happen? I can tell you myexample is that in the middle of the night is when these things usually start beeping saying thattheir battery is low, and you really don't want to service your smoke detector at that time.

Whatyou really want to do is make it stop beeping, which means you probably go up there and pull thebattery out of it. Your intentions were good. You're going to install that battery back in, but yousimply don't do it. So now you have a smoke detector with no means of alerting it.

The other thing that, you know, just finding the battery for a smoke detector is not without someamount of work. Like in our particular house, we have the battery drawer. It has a multitude ofbatteries in there. And because of our teenagers' activity and the way they treat batteries, an oldone is pulled out and a new one is put in, and the old one is dropped back into the drawer. Andso you have a whole collection of batteries that you're not sure of the capacity and such. And justremoving that aggravation would certainly be a benefit to the consumer.

But you can see; onceyou create these low and no-power applications and then connect them together with this low andno-power networking system, it creates a lot of opportunities to make the consumers' life much,much better.

* Another application is of Home automation(also called domotics) is the residential extension of "building automation". It is automation of the home, housework or household activity. Homeautomation may include centralized control of lighting, HVAC (heating, ventilation and airconditioning), appliances, and other systems, to provide improved convenience, comfort, energyefficiency and security.

Building automation optimizes energy savings and reduces operating costs by lowering total costof ownership. Essential to the success of building automation projects, self-powered wirelesstechnology gives architects, contractors and property owners the freedom to install all the sensorsthey need, of whatever type, wherever they like. Installation time is minimized and overall systemcosts are significantly reduced owing to 
device placement flexibility.With conventional battery-powered sensors, the cost of monitoring, replacing and recyclingbatteries increases as more units are installed.

Energy autonomous, battery-less sensors removethese headaches. The same advantages also apply to industrial plant installations. Having nobatteries means zero maintenance and the wireless technology creates new possibilities to locatesensors in previously inaccessible areas or remote storage vessels.

* EnOcean[28]is a wireless, energy harvesting technology used primarily in building automationsystems. It is not set out for international, European or national standardization;

However,EnOcean $\mathrm{GmbH}$ is offering its technology and licenses for the patented features under licensewithin the EnOcean Alliance framework. The concept was developed to enable battery lesssensors and switches for building automation.

Energy harvesting wireless sensors form EnOcean makes a home intelligent and energy-efficient.In residential building, you see an increasing number of houses that are striking not just for theirmodern architecture but also for the innovative, eco-friendly technology invested in them.

A majorobjective of this is energy efficiency.Self-powered wireless technology is ideal for retrofitting in residential building. If an attic isrenovated or expanded or further switches are to be added, EnOcean technology is the simple,speedy and value-for-money alternative to cabling, breaking and other time-consuming and costlyrenovation chores.

* There are also other applications like in efficient office energy control, surveillance and security, agricultural management etc as shown in figure 8 .
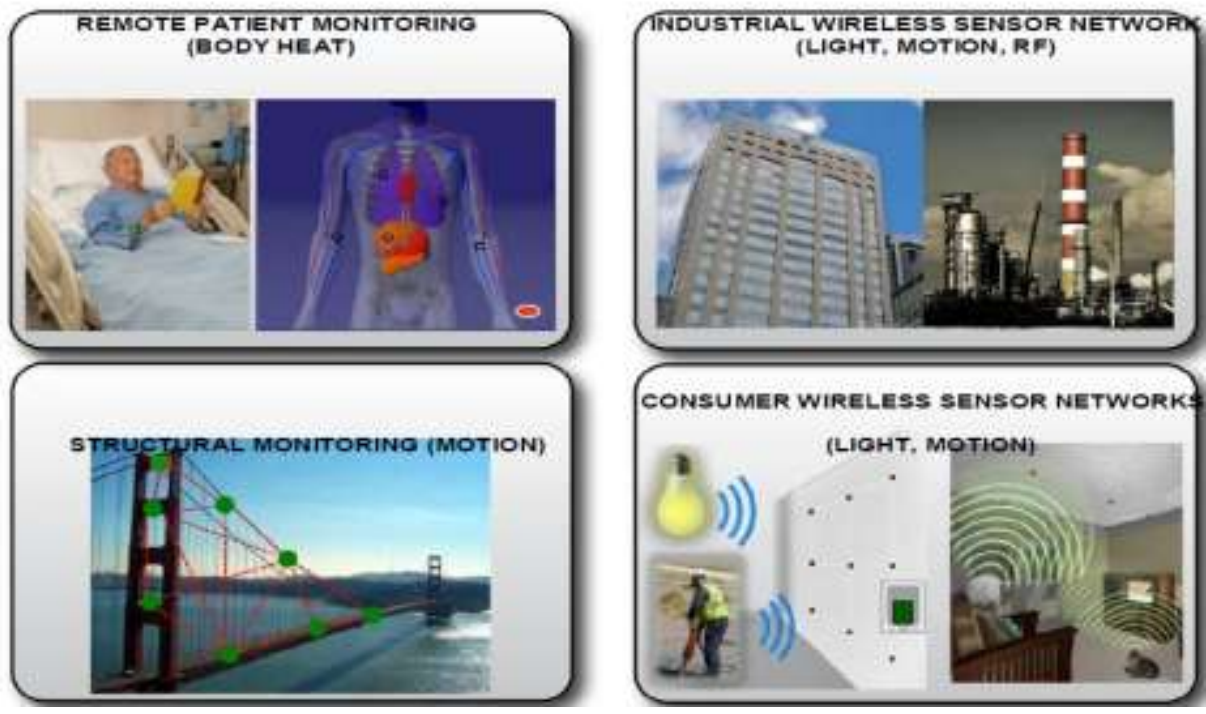

Figure 8 shows different applications of energy harvesting

\section{Conclusions}

While micro-energy harvesting may not generate a lot of power, what is created can be enough to offset, or even replace, the reliance on traditional power supplies. This is particularly true when the proper type of alternative energy is selected based on the environmental conditions to optimize generation.

The potential for piezoelectric energy-harvesting systems is great and spans many industries and sectors, including self-energizing MEMS; sensor and biomedical structural applications; and regenerative energy harvesting including vehicles, engineering structures, and household appliances.

The sustainable energy harvesting devices for engineering structures and household devices could ultimately be used to supply power to the grid. Material development and development of design approaches are currently being pursued to improve the capabilities for energy-harvesting devices.

\section{References}

[1] "Energy Harvesting" retrieved from- http://en.wikipedia.org/wiki/Energy harvesting.

[2] Jean-Claude Baumer, Director Strategic Marketing EMEA, "The Energy Challenge Smart SystemSolutions for Energy Efficiency" retrieved from Texas Instruments.

[3] Zigbee retrieved from- http://en.wikipedia.org/wiki/ZigBee.

[4] Blockdiagramretrievedfromhttp://www.ti.com/ww/en/apps/energyharvesting/index.shtml?DCMP=MSP430Energy\&HQS=Other+ OT+energyharvesting.

[5] MEMS retrieved from- http://en.wikipedia.org/wiki/Microelectromechanical_systems.

[6] JChad Skelton (7 FEB 2008): Knee-Mounted Device Generates Electricity while You Walk retrieved fromhttp://communities.canada.com/vancouversun/blogs/scienceinbc/archive/2008/02/07/revolutionary-knee-mounted-devicegenerated-electricity-while-you-walk.aspx. 
[7] Hausler.E, Stien.L (1 Dec1986):"ImplantablephysiologicalpowersupplywithPVDFfilm."Bibliography of Ferroelectrics Article (volume 70, pages 213-269) - Affiliation to Research Instituteof Electronics, Shizuka University, Hamamatsu, and Japan. Doi: $10.1080 / 00150198608221433$.

[8] Umdea M, Nakamura K, UehaS.(1996):"Analysisofthetransformationofmechanicalimpactenergyto electric energy using piezoelectric vibrator." Journal of Fiber Bioengineering andInformatics VOL. 1, NO.4 (2009). Doi:10.3993/jfbi03200902.

[9] Elvin NG,ElvinAA,SpectorM.(2001):"Aselfpoweredmechanicalstrainenergysensor."SmartMaterials and structures journal vol. 10, no. 2.Doi: $10.1088 / 0964-1726 / 10 / 2 / 314$.

[10] MichaelGrahamRichard,Ottawa,Canada(08.4.06):"Japan:ProducingElectricityfromTrainStationTicketGates"retrievedfromhttp://w ww.treehugger.com/files/2006/08/japan_ticket_gates.php.

[11] "Power leap" retrieved from http://www.powerleap.net/images/product.html.

[12] Dave White (August 4, 2006)"Commuter-generated electricity" retrieved from-http://www.gearlive.com/news/article/japanesescientists-working-on-commuter-generatedelectricity-0804061459.

[13] Shenck, N.S.; Paradiso, J.A. (May/Jun 2001) "Energy scavenging with shoe-mountedpiezoelectric," Micro, IEEE, vol.21, no.3, pp.30-42.

[14] Electro active polymers From Wikipediaretrievedfromhttp://en.wikipedia.org/wiki/Electroactive_polymers.

[15] "Innowattech" retrieved from http://www.innowattech.co.il/.

[16] Minnesota Department of Transportation retrieved from- http://www.dot.state.mn.us/.

[17] "Fuzzy Logic" retrieved from http://en.wikipedia.org/wiki/Fuzzy_logic.

[18] Sedona.et.al retrieved from http://www.yelp.com/topic/phoenix-sedona-et-al-touring-camping.

[19] Carnot cycle retrieved from http://en.wikipedia.org/wiki/Carnot_cycle.

[20] Thermoelectric generator retrieved from- http://en.wikipedia.org/wiki/Thermoelectric_generator.

[21] Tellurex Corporation retrieved from http://www.tellurex.com/.

[22] FraunhoferGesellschaft (8 February 2009) "Micropelt introduces world's first Thermo harvester-Driven Wireless Sensor Node enables battery-free operation of sensor nodes." retrieved from-http://www.micropelt.com/down/pm_te_power_node_engl.pdf.

[23] [23]M2EPowerKineticBattery(August22,2008)retrievedfromhttp://www.goodcleantech.com/2008/08/m2e_power_to_launch_batte ry_ch.php.

[24] KorneelRabaey, Nico Boon, Steven D. Siciliano, Marc Verhaege, and Willy Verstraete(September 2004) "biofuelcells". Applied and Environmental Microbiology journal, p. 5373-5382,Vol. 70, No. 9;0099-2240/04/\$08.00+0, DOI: 10.1128/AEM.70.9.53735382.2004 .

[25] The power within, by Bob Holmes, New Scientist, 25 August 2007.

[26] "Voltree's Website" Retrieved from http://voltreepower.com/index.html.

[27] Retrieved from The Energy Challenge Smart System Solutions for Energy Efficiency- Texasinstruments.

[28] Enocean retrieved from http://en.wikipedia.org/wiki/EnOcean. 